\title{
Inverse Optimal Adaptive Output Feedback Control of Euler-Lagrange Systems: A Variable Structure Observer Based Approach
}

\author{
Orhan Aksoy, Erkan Zergeroglu, and Enver Tatlicioglu
}

\begin{abstract}
This work focuses on inverse optimal, observer based output feedback control of Euler-Lagrange systems. Specifically a variable structure observer based output feedback controller is proposed which aside from ensuring asymptotic position tracking also ensures that a positive cost function, penalizing control input performance, is minimized. Simulation studies performed on a two link planar robot manipulator are included to illustrate the overall performance and feasibility of the proposed controller.
\end{abstract}

\section{INTRODUCTION}

Finding an optimal solution to the output feedback control of Euler-Lagrange systems is an extremely a challenging problem. For the "forward" optimal approach, one needs to solve the Hamilton-Jacobi-Bellman (HJB) equation, and finding a value function satisfying HJB equation for a nonlinear system is, even when all system states are measurable (the full-state feedback case), a challenging task. The inverse optimal approach on the other hand, is a constructive method in which one has to design a control-Lyapunov function, which is then treated as an optimal value function. By this way the overall problem is reduced to finding a cost function which minimizes the previously defined value function. Aside from finding a proper control-Lyapunov function, the inverse optimal approach is easier compared with the forward optimal approach as it does not involve the solution of HJB equation for a highly nonlinear system which may or may not exists. Some applications of inverse optimal controller approach to nonlinear systems can be found in [1]-[5].

For adaptive case, when the system parameters are uncertain, inverse optimal adaptive controllers such as [3], [4], [6][7] have been developed to compensate for the uncertainties that are linear in the parameters [2]. Results such as [3] and [7] proposed inverse optimal adaptive controllers for a general class of nonlinear systems with unknown parameters. An inverse optimal adaptive attitude tracking controller was proposed in [4] for rigid spacecraft with external disturbances and a constant uncertain inertia matrix. In [8], an inverse optimal adaptive backstepping technique is applied to the design of a pitch control law for a surface-to-air nonlinear

This research is supported by Grants of the Scientific and Technological Research Council of Turkey, TUBITAK Project No: 112E561.

O. Aksoy is with Havelsan A.S. Pendik, Istanbul, Turkey (Email: oaksoy@gmail.com)

E. Zergeroglu is with the Department of Computer Engineering, Gebze Technical University, 41400, Gebze, Kocaeli, Turkey (Email: ezerger@bilmuh.gyte.edu.tr).

E. Tatlicioglu is with the Department of Electrical \& Electronics Engineering, Izmir Institute of Technology, 35430, Urla, Izmir, Turkey (Email: enver@iyte.edu.tr). missile model with a constant inertia matrix [2]. In [1] and [2], the authors initially developed an inverse optimal adaptive controller for an Euler-Lagrange system with full state feedback, then extended their result to output feedback type using a nonlinear filter based approach.

In this study, via defining a positive cost function for the observer based adaptive output feedback controller proposed in [9], we have proved that under some conditions the proposed controller is optimal. Therefore the proposed controller not only ensures asymptotic tracking but it also ensures that a positive cost function, penalizing control input performance, is minimized.

The rest of the work is organized in the following manner: In Section II the dynamical model of the robot manipulator with its properties used in the analysis are presented. The formulation of the proposed observer-controller couple are presented Section III. Section IV presents the cost function and inverse optimal formulation. Simulation studies performed on a two link, direct drive planar robot manipulator are given in Section V. And finally concluding remarks are given in Section VI.

\section{System DyNAMICS AND PROPERTIES}

The nonlinear systems considered in this study are modeled by the following Euler-Lagrange formulation

$$
M(q) \ddot{q}+V_{m}(q, \dot{q}) \dot{q}+G(q)+F_{d} \dot{q}=\tau
$$

where $M(q) \in \mathbb{R}^{n \times n}$ represents the inertia matrix, $V_{m}(q, \dot{q}) \in \mathbb{R}^{n \times n}$ represents the centripetal-Coriolis matrix $G(q) \in \mathbb{R}^{n}$ represents the gravitational effects, $F_{d} \in \mathbb{R}^{n \times n}$ represents the friction, $\tau(t) \in \mathbb{R}^{n}$ represents the control input, $q(t), \dot{q}(t), \ddot{q}(t) \in \mathbb{R}^{n}$ represent the system state and its first and second time derivatives, respectively.

Euler-Lagrange systems exhibit the following properties that are used in the subsequent control development and analysis sections:

Property 1: The inertia matrix can be bounded from above and below by the following inequalities [12]

$$
m_{1} I_{n} \leq M(q) \leq m_{2} I_{n}
$$

where $m_{1}$ and $m_{2}$ are positive constants, and $I_{n}$ is the $n \times n$ identity matrix. Likewise the inverse of of the inertia matrix can be bounded as follows

$$
\frac{1}{m_{2}} I_{n} \leq M^{-1}(q) \leq \frac{1}{m_{1}} I_{n}
$$


Property 2: The inertia and the centripetal-Coriolis matrices satisfy the following relationship [10]

$$
\xi^{T}\left(\frac{1}{2} \dot{M}(q)-V_{m}(q, \dot{q})\right) \xi=0 \quad \forall \xi \in \mathbb{R}^{n}
$$

where $\dot{M}(q)$ represents the time derivative of the inertia matrix.

Property 3: The centripetal-Coriolis matrix satisfies the following relationship [11]

$$
V_{m}(q, \nu) \xi=V_{m}(q, \xi) \nu \quad \forall \xi, \nu \in \mathbb{R}^{n} .
$$

Property 4: The norm of the centripetal-Coriolis and friction matrices can be upper bounded as follows [12]

$$
\left\|V_{m}(q, \xi)\right\|_{i \infty} \leq \zeta_{c 1}\|\xi\|, \quad\left\|F_{d}\right\|_{i \infty} \leq \zeta_{f} \quad \forall \xi \in \mathbb{R}^{n} .
$$

where $\zeta_{c 1}$ and $\zeta_{f}$ are positive constants and $\|\cdot\|_{i \infty}$ denotes the matrix induced norm.

Property 5: The dynamics given in (1) can be linearly parameterized as follows [12]

$$
Y(q, \dot{q}, \ddot{q}) \theta=M(q) \ddot{q}+V_{m}(q, \dot{q}) \dot{q}+G(q)+F_{d} \dot{q}
$$

where $\theta \in \mathbb{R}^{p}$ contains the constant system parameters, $Y(q, \dot{q}, \ddot{q}) \in \mathbb{R}^{n \times p}$ denotes the regression matrix that is a function only of $q(t), \dot{q}(t)$ and $\ddot{q}(t)$. The formulation of (7) can also written in terms of the desired trajectory in the following manner

$$
Y_{d}\left(q_{d}, \dot{q}_{d}, \ddot{q}_{d}\right) \theta=M\left(q_{d}\right) \ddot{q}_{d}+V_{m}\left(q_{d}, \dot{q}_{d}\right) \dot{q}_{d}+G\left(q_{d}\right)+F_{d} \dot{q}_{d}
$$

where the desired regression matrix $Y_{d}\left(q_{d}, \dot{q}_{d}, \ddot{q}_{d}\right) \in \mathbb{R}^{n \times p}$ is a function of the desired system state, its first and second time derivatives denoted by $q_{d}(t), \dot{q}_{d}(t), \ddot{q}_{d}(t) \in \mathbb{R}^{n}$, respectively.

Property 6: The inertia, centripetal-Coriolis, and gravity terms of (1) can be upper bounded as follows [13]

$$
\begin{aligned}
\|M(\xi)-M(\nu)\|_{i \infty} & \leq \zeta_{m 1}\|\xi-\nu\| \\
\left\|M^{-1}(\xi)-M^{-1}(\nu)\right\|_{i \infty} & \leq \zeta_{m 2}\|\xi-\nu\| \\
\left\|V_{m}(\xi, \omega)-V_{m}(\nu, \omega)\right\|_{i \infty} & \leq \zeta_{c 2}\|\omega\|\|\xi-\nu\| \\
\|G(\xi)-G(\nu)\| & \leq \zeta_{g}\|\xi-\nu\|
\end{aligned}
$$

where $\nu, \xi, \omega \in \mathbb{R}^{n}, \zeta_{m 1}, \zeta_{m 2}, \zeta_{c 2}, \zeta_{g} \in \mathbb{R}$ are positive bounding constants.

\section{AdAPtive OBSERVER BASED CONTROLler}

The control objective is to design an adaptive tracking controller for the system using only $q(t)$ as the available system state, despite the presence of uncertain system parameters while minimizing a meaningful performance index. Keeping all the signals under the closed-loop operation is the hidden boundedness objective.

We will quantify the control objective by defining the link position tracking error $e(t) \in \mathbb{R}^{n}$ as follows

$$
e \triangleq q_{d}-q
$$

It is noted that the desired system state $q_{d}(t)$ and its derivatives are required to be chosen as bounded functions of time.
In order to compensate the uncertainty of the system parameters, a dynamic adaptive estimate of the system parameters, denoted by $\hat{\theta}(t) \in \mathbb{R}^{p}$, is developed in the subsequent sections. The difference between the model parameter vector $\theta$ and estimated parameters is defined as

$$
\tilde{\theta} \triangleq \theta-\hat{\theta}
$$

where $\tilde{\theta}(t) \in \mathbb{R}^{p}$ is the parameter estimation error vector.

Since, $\dot{q}$ measurements are not available for control design, an observer for velocity, denoted by $\dot{\hat{q}}(t) \in \mathbb{R}^{n}$, is utilized in the design of the subsequent controller. The corresponding velocity and position observation errors, $\dot{\tilde{q}}(t), \tilde{q}(t) \in \mathbb{R}^{n}$ are defined as

$$
\begin{aligned}
& \dot{\tilde{q}} \triangleq \dot{q}-\dot{\hat{q}} \\
& \tilde{q} \triangleq q-\hat{q} .
\end{aligned}
$$

The following model-free velocity observer described in [9] is utilized in the controller

$$
\begin{aligned}
& \dot{\hat{q}}=p+K_{0} \tilde{q}-K_{c} e \\
& \dot{p}=K_{1} \operatorname{Sgn}(\tilde{q})+K_{2} \tilde{q}-\alpha K_{c} e
\end{aligned}
$$

where $p(t) \in \mathbb{R}^{n}$ is an auxiliary filter variable, $K_{0}, K_{c}, K_{1}$, $K_{2} \in \mathbb{R}^{n \times n}$ are positive definite, diagonal gain matrices, $\alpha \in \mathbb{R}$ is a positive gain, and $\operatorname{Sgn}(\tilde{q}) \in \mathbb{R}^{n}$ is the vector form of the standard signum function which has the following form

$$
\operatorname{Sgn}(\tilde{q}) \triangleq\left[\operatorname{sgn}\left(\tilde{q}_{1}\right) \quad \operatorname{sgn}\left(\tilde{q}_{2}\right) \quad \ldots \quad \operatorname{sgn}\left(\tilde{q}_{n}\right)\right]^{T} .
$$

To avoid second order time derivatives in the subsequent stability analysis, a filtered tracking error, denoted by $r(t) \in$ $\mathbb{R}^{n}$, and a filtered version of the observation error, denoted by $s(t) \in \mathbb{R}^{n}$, are formulated as

$$
r \triangleq \dot{e}+\alpha e \text { and } s \triangleq \dot{\tilde{q}}+\alpha \tilde{q} .
$$

In order to facilitate the development of the inverse optimal controller formulation, the input is segregated into two parts. First one is the feedforward component of the control input, denoted by $u_{f}(t) \in \mathbb{R}^{n}$, which is independent of the system states, and the second one is the optimal feedback law, denoted by $u_{0}(t) \in \mathbb{R}^{n}$, which depends on the system states. In view of this segregation, the control input is designed as

$$
\tau=u_{f}-u_{0}
$$

with feedforward and optimal components of the control input designed as

$$
\begin{aligned}
& u_{f}=Y_{d} \hat{\theta} \\
& u_{0}=-\left(K_{p} e+\alpha K_{c}\left(q_{d}-\hat{q}\right)+K_{c}\left(\dot{q}_{d}-\dot{\hat{q}}\right)\right)
\end{aligned}
$$

where $K_{p} \in \mathbb{R}^{n \times n}$ is a diagonal, positive definite gain matrix. The dynamic adaptive estimate for the uncertain model parameter vector is generated according to

$$
\begin{aligned}
\hat{\theta}= & \Gamma \int_{0}^{t}\left[\alpha Y_{d}^{T}(\sigma) e(\sigma)-\dot{Y}_{d}^{T}(\sigma) e(\sigma)\right] d \sigma \\
& +\Gamma Y_{d}^{T}(t) e(t)-\Gamma Y_{d}^{T}(0) e(0)
\end{aligned}
$$


where $\Gamma \in \mathbb{R}^{p \times p}$ is a constant, diagonal, positive definite adaptation gain matrix. Using the time derivative of the observer dynamics in (14), system dynamics in (1), desired system dynamics in (7) and the control input designed in (18), (19), (20), the observation error dynamics is obtained as

$$
\ddot{\tilde{q}}=N_{0}-K_{1} \operatorname{Sgn}(\tilde{q})-K_{2} \tilde{q}-K_{0} \dot{\tilde{q}}+K_{c} r
$$

where $N_{0}(t) \in \mathbb{R}^{n}$ is an auxiliary term defined as

$$
N_{0}=M^{-1}(q)\left\{\tau-V_{m}(q, \dot{q}) \dot{q}-G(q)-F_{d} \dot{q}\right\} .
$$

The above auxiliary term can be segregated as sum of two auxiliary terms, denoted by $N_{d}(t) \in \mathbb{R}^{n}$ and $N_{b}(t) \in \mathbb{R}^{n}$, that can be bounded by a constant and by a function of error signals. Specifically,

$$
N_{0}=N_{d}+N_{b}
$$

where $N_{b}$ can be bounded as [9]

$$
\left\|N_{b}\right\| \leq \rho_{01}\|e\|+\rho_{02}\|r\|+\rho_{03}\|r\|^{2}+\rho_{04}\|s\|
$$

where $\rho_{01}, \rho_{02}, \rho_{03}, \rho_{04} \in \mathbb{R}$ are known positive bounding constants and the entries of $N_{d}$ and its first time derivative can be bounded in the following manner

$$
\left\|N_{d i}(t)\right\|_{\mathcal{L}_{\infty}}<\zeta_{N_{d i}},\left\|\dot{N}_{d i}(t)\right\|_{\mathcal{L}_{\infty}}<\zeta_{\dot{N}_{d i}}
$$

where subscript $i$ denotes the $i^{\text {th }}$ entry of the vector, and $\zeta_{N_{d i}}, \zeta_{\dot{N}_{d i}} \in \mathbb{R} i=1, \cdots, n$ are known positive bounding constants.

When the observer gains are selected to satisfy

$$
\alpha\left(K_{0}-\alpha I_{n}\right)=K_{2}
$$

then, the filtered observer error dynamics is obtained as

$$
\dot{s}=N_{d}+N_{b}-K_{1} \operatorname{Sgn}(\tilde{q})-\frac{K_{2}}{\alpha} s+K_{c} r .
$$

The filtered error dynamics is derived using (1) along with the time derivative of the filtered tracking error in (17)

$$
M(q) \dot{r}=-V_{m}(q, \dot{q}) r-K_{p} e-K_{c} r-K_{c} s+\varphi+Y_{d} \tilde{\theta}
$$

where the control input in (18), (19), (20) is substituted and $\varphi(t) \in \mathbb{R}^{n}$ is an auxiliary error-like term defined as

$$
\varphi \triangleq Y_{s} \theta-Y_{d} \theta
$$

where $Y_{s}(t) \in \mathbb{R}^{n \times p}$ is obtained from the following expression

$$
Y_{s} \theta=M(q)\left(\ddot{q}_{d}+\alpha \dot{e}\right)+V_{m}(q, \dot{q})\left(\dot{q}_{d}+\alpha e\right)+G(q)+F_{d} \dot{q} .
$$

The auxiliary error-like term in (30) can be bounded as

$$
\|\varphi\| \leq \rho_{1}(\|e\|)\|e\|+\rho_{2}(\|e\|)\|r\|
$$

where $\rho_{1}(\|e\|), \rho_{2}(\|e\|) \in \mathbb{R}$ are known positive bounding functions.

Having obtained closed-loop error dynamics for both filtered tracking error and filtered observation error, we are now ready to proceed with the stability analysis.
Theorem 1: The controller given in (18) and observer given in (14) ensures semi-global asymptotic stability in the sense that $\|e(t)\|,\|\dot{\tilde{q}}(t)\| \rightarrow 0$ as $t \rightarrow \infty$ provided that the gains are selected to satisfy and (27), and controller gain $K_{c}$, and observer gains $K_{2}$ and $K_{1}$ are chosen to satisfy

$$
\begin{aligned}
K_{c} & =1+\rho_{2}+k_{n} \rho_{1}^{2} \\
K_{2} & =\alpha\left(1+\rho_{04}+k_{n}\left(\rho_{01}^{2}+\rho_{02}^{2}+\rho_{03}^{2}\right)\right) \\
K_{1 i} & >\zeta_{N_{d i}}+\frac{1}{\alpha} \zeta_{\dot{N}_{d i}}
\end{aligned}
$$

where $k_{n} \in \mathbb{R}$ is a nonlinear damping gain which needs to be selected to satisfy

$$
k_{n}>\frac{1}{2}\left(1+\frac{\lambda_{2}}{\lambda_{1}}\|z(0)\|^{2}\right) .
$$

In (36), $z(t) \in \mathbb{R}^{(3 n+p+1) \times 1}$ is defined as

$$
z=\left[s^{T}, \sqrt{P_{0}}, r^{T}, e^{T}, \tilde{\theta}^{T}\right]^{T}
$$

and $\lambda_{1}, \lambda_{2} \in \mathbb{R}$ are defined as

$$
\begin{aligned}
& \lambda_{1}=\frac{1}{2} \min \left\{1, m_{1}, \lambda_{\min }\left(K_{p}\right), \lambda_{\min }\left(\Gamma^{-1}\right)\right\} \\
& \lambda_{2}=\frac{1}{2} \max \left\{1, m_{2}, \lambda_{\max }\left(K_{p}\right), \lambda_{\max }\left(\Gamma^{-1}\right)\right\} .
\end{aligned}
$$

Proof: To prove the theorem, the following scalar function $V(z) \in \mathbb{R}$ is defined as

$$
V=\frac{1}{2} s^{T} s+P_{0}+\frac{1}{2} r^{T} M(q) r+\frac{1}{2} e^{T} K_{p} e+\frac{1}{2} \tilde{\theta}^{T} \Gamma^{-1} \tilde{\theta}
$$

where the scalar auxiliary function $P_{0}(t) \in \mathbb{R}$ is defined as

$$
P_{0} \triangleq \zeta_{0}-\int_{0}^{t} \omega_{0}(\sigma) d \sigma
$$

where $\omega_{0}(t) \in \mathbb{R}$ and non-negative constant $\zeta_{0}$ are defined as

$$
\begin{aligned}
\omega_{0}(t) & \triangleq s^{T}\left(N_{d}-K_{1} \operatorname{Sgn}(\tilde{q})\right) \\
\zeta_{0} & \triangleq \sum_{i=1}^{n} K_{1 i}\left|\tilde{q}_{i}(0)\right|-\tilde{q}^{T}(0) N_{d}(0) .
\end{aligned}
$$

The axuiliary term $P_{0}(t)$ in (40) can be proven to be nonnegative provided that the entries of the observer gain matrix $K_{1}$ are chosen to satisfy (35) as demonstrated in [9].

The Lyapunov function in (40) can be lower and upper in the following sense

$$
\lambda_{1}\|x\|^{2} \leq \lambda_{1}\|z\|^{2} \leq V \leq \lambda_{2}\|z\|^{2}
$$

where $x(t) \in \mathbb{R}^{3 n}$ is defined as

$$
x=\left[\begin{array}{lll}
s^{T} & r^{T} & e^{T}
\end{array}\right]^{T} .
$$

Using the filtered error dynamics in (29), filtered observer error dynamics in (28), the time derivative of the parameter update law in (21) and properties (2)-(6) along with the time derivative of the Lyapunov function in (40) results in

$$
\dot{V}=s^{T}\left(N_{b}-\frac{K_{2}}{\alpha} s\right)+r^{T}\left(\varphi-K_{c} r\right)-\alpha e^{T} K_{p} e .
$$


Using bounds in (25) and (32), we can obtain the following upper bound for the right hand side of the above expression

$$
\begin{aligned}
\dot{V} \leq & -\|s\|^{2}-\|r\|^{2}-\|e\|^{2} \\
& +\left[\rho_{01}\|s\|\|e\|-k_{n} \rho_{01}^{2}\|s\|^{2}\right] \\
& +\left[\rho_{02}\|s\|\|r\|-k_{n} \rho_{02}^{2}\|s\|^{2}\right] \\
& +\left[\rho_{03}\|s\|\|r\|^{2}-k_{n} \rho_{03}^{2}\|s\|^{2}\right] \\
& +\left[\rho_{1}\|r\|\|e\|-k_{n} \rho_{1}^{2}\|r\|^{2}\right] .
\end{aligned}
$$

Completing the squares for the terms in the brackets, and using definition (45), we can reach the following upper bound for the right hand side of the time derivative of the Lyapunov function

$$
\dot{V} \leq-\left[1-\frac{1}{2 k_{n}}\left(1+\|x\|^{2}\right)\right]\|x\|^{2}
$$

where $\alpha \Lambda_{\min }\left(K_{p}\right)>1$ was also considered. Provided that (36) is satisfied, the right hand side of the above expression can be upper bounded as

$$
\dot{V} \leq-\beta\|x\|^{2}
$$

where $\beta \in \mathbb{R}$ is some positive constant satisfying $0<\beta \leq 1$.

In view of (40) and (49), $V(z)$ and thus $z(t) \in \mathcal{L}_{\infty}$. Using standard signal chasing arguments, it can be shown that all closed-loop signals including $e(t), \dot{e}(t)$ and $\dot{\tilde{q}}(t)$, $\ddot{\tilde{q}}(t)$ are bounded. By integrating both sides of (49) in time from initial time to infinity, it is easy to see that $x(t) \in \mathcal{L}_{2}$, therefore $e(t), \dot{\tilde{q}}(t) \in \mathcal{L}_{2}$. Finally, using Barbalat's Lemma, it is proven that $\|e(t)\|,\|\dot{\tilde{q}}(t)\| \rightarrow 0$ as $t \rightarrow \infty$.

\section{Cost Functional Minimization}

In this section, the minimization of a subsequently chosen cost functional is demonstrated. The following theorem encapsulate the proof of the minimization.

Theorem 2: The output feedback law in (19) and the adaptive update law in (21) minimizes the following positive cost functional

$J=\lim _{t \rightarrow \infty}\left\{\frac{k_{A}}{2} \tilde{\theta}^{T} \Gamma^{-1} \tilde{\theta}+\int_{0}^{t}\left[l(x(\sigma))+u_{0}^{T}(\sigma) R u_{0}(\sigma)\right] d \sigma\right\}$

and $l(x) \in \mathbb{R}$ is defined by

$$
l=-k_{A} \dot{V}-u_{0}^{T} R u_{0}
$$

where $V$ is the Lyapunov function defined in (40), $R \in \mathbb{R}^{n \times n}$ is a positive definite, symmetric weighting matrix, and $k_{A} \in$ $\mathbb{R}$ is a positive constant satisfying

$$
k_{A} \geq \frac{1}{\beta} \lambda_{\max }\left(K_{g}^{T} R K_{g}\right)
$$

where $K_{g} \in \mathbb{R}^{n \times 3 n}$ is defined by

$$
K_{g} \triangleq\left[\begin{array}{lll}
K_{c} & K_{c} & K_{p}
\end{array}\right]
$$

Proof: We would like to note that for the cost function in (50) to be considered meaningful, it has to be a positive function of the states $x$ and the input $u_{0}$. To prove that the term $u_{0}^{T} R u_{0}$ positive, rewrite the input $u_{0}$ in the following form

$$
u_{0}=-K_{g} x
$$

which results in

$$
u_{0}^{T} R u_{0}=x^{T} K_{g}^{T} R K_{g} x
$$

which is positive if $K_{g}^{T} R K_{g}$ is positive definite. Since we select $R, K_{c}$ and $K_{p}$ as positive definite gain matrices, this requirement is satisfied.

To prove that the function $l(x)$ is positive, (51) and (55) are utilized to obtain

$$
l=-k_{A} \dot{V}-x^{T} K_{g}^{T} R K_{g} x .
$$

The expression in (49) can be rewritten as

$$
-k_{A} \dot{V} \geq k_{A} \beta x^{T} x .
$$

Adding and subtracting the term $x^{T} K_{g}^{T} R K_{g} x$ to the right hand side of (57) allows us to find a lower bound for the right hand side of (56) as

$$
x^{T}\left(k_{A} \beta I_{n}-K_{g}^{T} R K_{g}\right) x \geq 0
$$

from which it is easy to conclude that $l(x)$ is positive provided that $k_{A}$ satisfies (52). So, it is proved that for a positive definite and symmetric weighting matrix $R$ the cost function $J$ in (50) is a positive cost penalizing $e, r$ and $s$ if $k_{A}$ satisfies (52).

To show that $u_{0}$ minimizes $J$, the auxiliary input signal $v \in \mathbb{R}^{n}$ is defined

$$
v=u_{0}+K_{g} x
$$

Substituting this auxiliary input in the cost functional in (50) yields

$$
J=\lim _{t \rightarrow \infty}\left\{\frac{k_{A}}{2} \tilde{\theta}^{T} \Gamma^{-1} \tilde{\theta}+\int_{0}^{t}\left(-k_{A} \dot{V}+v^{T} R v\right) d s\right\} .
$$

Separating the parameter error cost from $V$ yields

$$
J=\lim _{t \rightarrow \infty}\left\{\begin{array}{c}
\frac{k_{A}}{2} \tilde{\theta}^{T} \Gamma^{-1} \tilde{\theta}-\frac{k_{A}}{2} \int_{0}^{t} \frac{d}{d t}\left(\tilde{\theta}^{T} \Gamma^{-1} \tilde{\theta}\right) d s \\
+k_{A}^{t} \int\left(-\dot{V}_{a}\right) d s+\int_{0}^{t}\left(v^{T} R v\right) d s
\end{array}\right\}
$$

where $V_{a} \in \mathbb{R}$ is defined by

$$
V_{a}=P_{0}+\frac{1}{2} s^{T} s+\frac{1}{2} r^{T} M(q) r+\frac{1}{2} e^{T} K_{p} e .
$$

Then, calculating the integrals in (61) gives

$J=\frac{k_{A}}{2} \tilde{\theta}(0)^{T} \Gamma^{-1} \tilde{\theta}(0)+k_{A} V_{a}(0)-k_{A} V_{a}(\infty)+\int_{0}^{\infty}\left(v^{T} R v\right) d s$.

Since from the proof of Theorem 1, we know that $x(t)$ converges to zero, then it is easy to conclude that the cost functional in (50) is minimized if $v=0$. 


\section{Simulation Studies}

The performance of the controller is examined in a numerical simulation on a two-link, direct-drive planar robot manipulator having the following dynamic model

$$
\begin{aligned}
M(q) & =\left[\begin{array}{cc}
p_{1}+2 p_{3} \cos \left(q_{2}\right) & p_{2}+p_{3} \cos \left(q_{2}\right) \\
p_{2}+p_{3} \cos \left(q_{2}\right) & p_{2}
\end{array}\right](64) \\
V_{m}(q, \dot{q}) & =\left[\begin{array}{cc}
-p_{3} \sin \left(q_{2}\right) \dot{q}_{2} & -p_{3} \sin \left(q_{2}\right)\left(\dot{q}_{1}+\dot{q}_{2}\right) \\
p_{3} \sin \left(q_{2}\right) \dot{q}_{1} & 0
\end{array}\right] \\
F_{d} & =\left[\begin{array}{cc}
f_{d 1} & 0 \\
0 & f_{d 2}
\end{array}\right], G(q)=\left[\begin{array}{cc}
0 & 0 \\
0 & 0
\end{array}\right]
\end{aligned}
$$

where $p_{1}=3.473\left[\mathrm{~kg}-\mathrm{m}^{2}\right], p_{2}=0.193\left[\mathrm{~kg}-\mathrm{m}^{2}\right]$, $p_{3}=0.242\left[\mathrm{~kg}-\mathrm{m}^{2}\right], f_{d 1}=5.3[\mathrm{Nm}-\mathrm{sec}], f_{d 2}=1.1$ $[\mathrm{Nm}-\mathrm{sec}]$. The desired trajectory is given as

$$
q_{d}(t)=\left[\begin{array}{l}
0.7 \sin (t)\left(1-\exp \left(-0.3 t^{3}\right)\right) \\
1.2 \sin (t)\left(1-\exp \left(-0.3 t^{3}\right)\right)
\end{array}\right][\mathrm{rad}]
$$

The control gains were selected as

$$
\begin{aligned}
\alpha & =\operatorname{diag}\{1.8,1.6\} \\
K_{p} & =\operatorname{diag}\{80,75\}, K_{c}=\operatorname{diag}\{6,2\} \\
K_{1} & =\operatorname{diag}\{1.6,1.2\}, K_{o}=\operatorname{diag}\{8,6\}
\end{aligned}
$$

The tracking errors and control torques are shown in Figures 1 and 2, respectively. The estimates for parameters are shown on Figure 3 and the cost value for $k_{A}=200$ is shown on Figure 4. From Figure 1, it can be concluded that the tacking control objective is achieved.
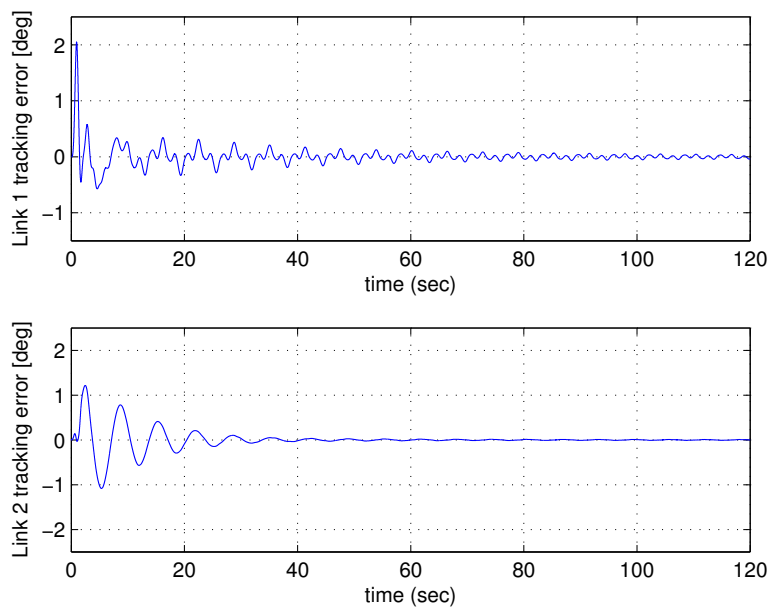

Fig. 1. Link Tracking Errors

\section{CONCLUSION}

It is shown that the observer-controller described in [9] not only tracks a time varying trajectory asymptotically, but also minimizes a positive cost function using only output information and parametric uncertainty. The condition for inverse optimality is derived, and simulation results are presented for a two link planar robot model.
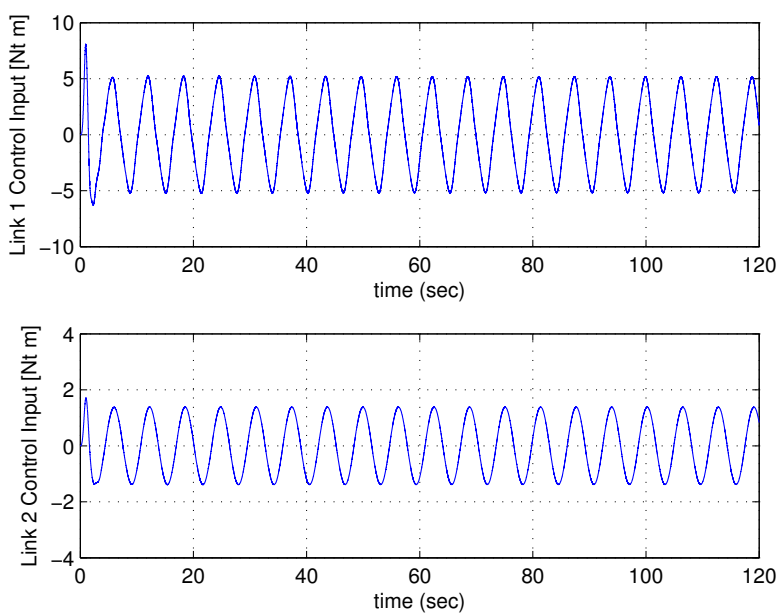

Fig. 2. Control input Torques

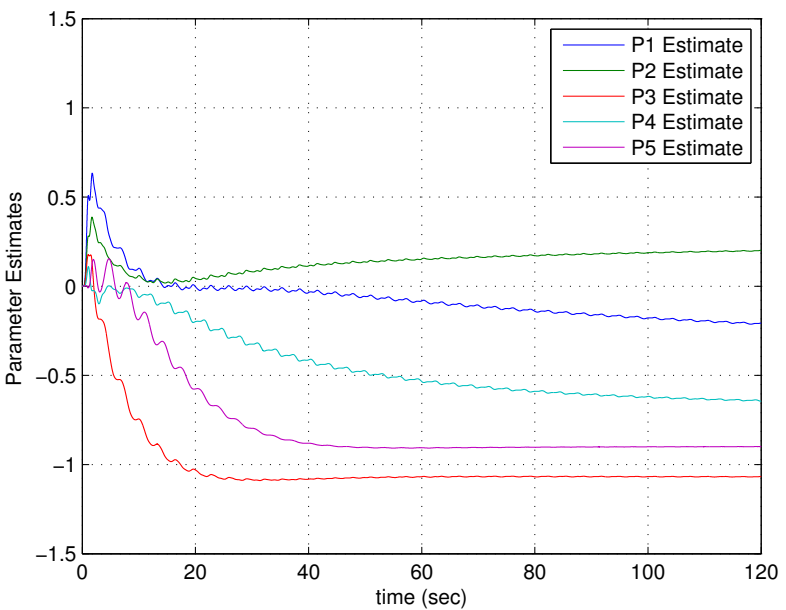

Fig. 3. Parameter estimates

\section{REFERENCES}

[1] K. Dupree, P . M. Patre, M. Johnson, and W . E. Dixon, "Inverse Optimal Adaptive Control of a Nonlinear Euler-Lagrange System, Part I: Full State Feedback", 48th IEEE Conference on Decision and Control, pp. 321-326, 2009.

[2] K. Dupree, M. Johnson, P . M. Patre, and W . E. Dixon, "Inverse Optimal Adaptive Control of a Nonlinear Euler-Lagrange System, Part II: Output Feedback", 48th IEEE Conference on Decision and Control, pp. 327-332, 2009.

[3] Z. Li and M. Krstic,"Optimal design of adaptive tracking controllers for nonlinear systems", Automatica, vol. 33, no. 4, pp. 1459-1473, 1997.

[4] W. Luo, Y . Chu, and K. Ling, "Inverse optimal adaptive control for attitude tracking of spacecraft, "IEEE Transactions on Automatic Control, vol. 50, no. 11, pp. 1639-1654, Nov . 2005.

[5] M. Krstic and P . V . Kokotovic, "Control lyapunov functions for adaptive nonlinear stabilization, "Systems and Control Letters,vol.26, no. 1, pp. 17-23, 1995.

[6] J. L. Fausz, V -S. Chellaboina, and W. Haddad, "Inverse optimal adaptive control for nonlinear uncertain systems with exogenous disturbances, " International Journal of Adaptive Control and Signal Processing, vol. 14, no. 1, pp. 1-38, 2000.

[7] X.-S. Cai and Z.-Z. Han, "Inverse optimal control of nonlinear systems 


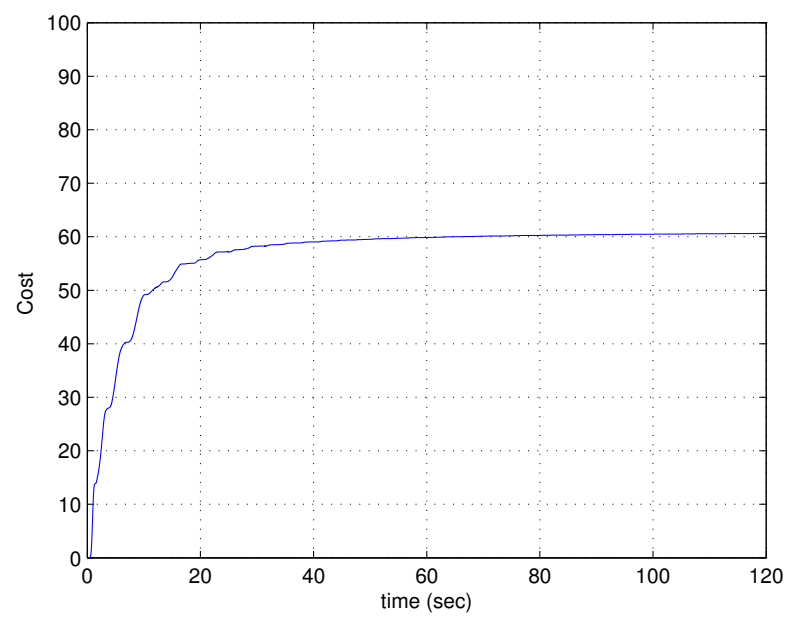

Fig. 4. Cost Value for $k_{A}=200$

with structural uncertainty ," IEE Proceedings of Contr ol Theory and Applications, vol. 152, no. 1, pp. 79-84, 2005.

[8] L. Sonneveldt,E.V an Oort,Q. P .Chu,and J. A. Mulder,"Comparison of inverse optimal and tuning functions designs for adaptive missile control, " Journal of Guidance, Control, and Dynamics,vol.31, no.4, pp. 1176-1182, 2008.

[9] E. Zergeroglu, Enver Tatlicioglu, "Observer Based Adaptive Output Feedback Tracking Control of Robot Manipulators", 49th IEEE Conference on Decision and Control, pp. 3638-3643, Atlanta, USA, December 15-17, 2010.

[10] P. Tomei, "Adaptive PD Controller for Robot Manipulators", IEEE Transactions on Robotics and Automation, Vol. 7, No. 4, pp. 565-570, August 1991.

[11] S. Nicosia and P. Tomei, "Robot Control by Using Only Position Measurements", IEEE Trans. Automatic Control, Vol. 35, No. 9, pp. 1058-1061, Sept. 1990.

[12] F.L. Lewis, C.T. Abdallah, and D.M. Dawson, Control of Robot Manipulators, New York: Macmillan Publishing Co., 1993..

[13] N. Sadegh, and R. Horowitz, "Stability and Robustness Analysis of a Class of Adaptive Controllers for Robot Manipulators", Int. J. Robotics Research, Vol. 9, No. 3, pp. 74-92, 1990. 